\title{
Carboplatin plus paclitaxel in combination with bevacizumab for the treatment of adenocarcinoma with interstitial lung diseases
}

\author{
HIDEKAZU SUZUKI, TOMONORI HIRASHIMA, MASASHI KOBAYASHI, NORIO OKAMOTO, \\ YUKA MATSUURA, MOTOHIRO TAMIYA, NAOKO MORISHITA, KOHEI OKAFUJI, \\ TAKAYUKI SHIROYAMA, OSAMU MORIMURA, SATOMU MORITA and ICHIRO KAWASE \\ Department of Thoracic Malignancy, Osaka Prefectural Medical Center for \\ Respiratory and Allergic Diseases, Osaka 583-8588, Japan
}

Received July 26, 2012; Accepted January 9, 2013

DOI: $10.3892 / \mathrm{mco} .2013 .82$

\begin{abstract}
Interstitial lung diseases (ILDs) are frequently associated with lung cancer. The safety of carboplatin plus paclitaxel in combination with bevacizumab (CP-B) in patients with ILD and lung cancer (ILD-LC) remains to be clarified. In the present study, the safety and efficacy of CP-B treatment in ILD-LC patients were retrospectively investigated. Four patients, who completed CP-B therapy, were included in this study. The dose of carboplatin was the area under the curve 5 , paclitaxel was $200 \mathrm{mg} / \mathrm{m}^{2}$ and bevacizumab was $15 \mathrm{mg} / \mathrm{kg}$ at treatment initiation. The patients were males, had histologically confirmed adenocarcinoma, were smokers and demonstrated non-usual interstitial pneumonia (non-UIP) patterns on computed tomography (CT). Patients received 1-6 cycles of CP-B therapy. Three of the four patients received maintenance bevacizumab therapy for 3-10 cycles. Only one patient demonstrated a partial response. Neutropenia was the most frequent adverse event. One patient experienced gut perforation during the first course of CP-B. No pulmonary toxicity was observed. Thus, treatment of ILD-LC patients with CP-B was not associated with pulmonary toxicity, however, this study population appeared to be at a low risk.
\end{abstract}

\section{Introduction}

Interstitial lung diseases (ILDs) with lung cancer (ILD-LC) have been reported in 9.8-38\% of ILD patients (1). There is no standard treatment for ILD-LC, regardless of whether small cell lung cancer (SCLC) or non-small cell lung cancer

Correspondence to: Dr Hidekazu Suzuki, Department of Thoracic Malignancy, Osaka Prefectural Medical Center for Respiratory and Allergic Diseases, 3-7-1 Habikino, Habikino-shi, Osaka 583-8588, Japan

E-mail: suzukih@ra.opho.jp

Key words: bevacizumab, interstitial lung disease, lung cancer, pneumonitis
(NSCLC) is diagnosed. Surgical resection and anticancer agents are associated with a high risk of ILD exacerbation (2). In patients with advanced ILD-NSCLC, carboplatin plus paclitaxel (CP) therapy is a common treatment option. However, a prospective study showed that $5.6 \%$ of patients experience acute exacerbation of ILD-NSCLC with CP therapy (3).

Findings of a clinical trial showed that the addition of bevacizumab to CP (CP-B) improved the overall survival in patients with advanced non-squamous cell NSCLC (4). This drug regimen is therefore considered a standard treatment option for this patient population. However, the safety of CP-B in patients with ILD-NSCLC remains to be clarified, especially with respect to lung toxicity. In the present study, the safety and efficacy of CP-B therapy in ILD-NSCLC patients were retrospectively investigated.

\section{Patients and methods}

Patients. ILD-NSCLC patients who newly received CP-B therapy between January, 2010 and December, 2011 at the Osaka Prefectural Medical Center for Respiratory and Allergic Diseases were retrospectively investigated based on their medical records. This study was approved by the Institutional Review Board. Five patients initially received CP-B therapy between January, 2010 and December, 2011. The four patients (male; age range 69-70 years) who completed the CP-B treatment were included in this study. Three of the four patients were smokers. These patients did not have brain metastases, a history of hemoptysis or an easy to bleed tumor at the initiation of the CP-B treatment.

Methods. ILD was identified by conventional or highresolution computed tomography (CT) images obtained prior to chemotherapy. Where CT images showed bilateral honeycomb, reticular or grand-glass opacities superior in the subpleural areas of both lungs, ILD was confirmed. Usual interstitial pneumonia (UIP) patterns were defined based on the presence of traction bronchiectasis and subpleural honeycombing. No bronchoalveolar lavage or lung biopsy were performed to confirm interstitial pneumonia. In addition, patients with a history of exposure to dust, such as asbestos, and those with collagen diseases were not excluded. Response 
Table I. Patient characteristics.

\begin{tabular}{lclllllllrr}
\hline Case & Age (years) & P-Y & His & ILD pattern & PS & Cyc & Main & Res & Reason & TTF (days) \\
\hline 1 & 69 & 76.5 & Ad & Non-UIP & 2 & 1 & - & NE & Toxicity \\
2 & 69 & 17 & Ad & Non-UIP & 1 & 6 & 6 & PR & PD & 281 \\
3 & 71 & 37 & Ad & Non-UIP & 0 & 4 & 3 & SD & PD & 200 \\
4 & 70 & 50 & Ad & Non-UIP & 1 & 4 & 10 & SD & PD & 487 \\
\hline
\end{tabular}

P-Y, pack-year; His, histology of lung cancer; Ad, adenocarcinoma; ILD, interstitial lung disease; UIP, usual interstitial pneumonia; PS, performance status; Cyc, cycles of carboplatin + paclitaxel + bevacizumab; Main, maintenance cycles of bevacizumab; Res, response of treatment; NE, not evaluated; PR, partial response; SD, stable disease; Reason, reason treatment was terminated; PD, progressive disease; TTP, time to treatment failure.

Table II. Treatment-related toxicity.

\begin{tabular}{lcccccc}
\hline Case & Neu & Hgb & Plt & U-prot & HpT & Others \\
\hline 1 & - & - & - & - & - & G4 gut perforation \\
2 & G4 & - & - & G2 & - & \\
3 & G4 & G2 & G2 & - & - & G2 infection \\
4 & G3 & G2 & - & - & G2 & \\
\hline
\end{tabular}

Neu, neutropenia; G, grade; Hgb, hemoglobin; Plt, platelet decrease; U-prot, urinary protein; HpT, hypertension.

Evaluation Criteria in solid tumors (5) were used for the evaluation of treatment response. Adverse events were evaluated using the Common Terminology Criteria for Adverse Events version 4.0. The time period to treatment failure was measured as the period between diagnosis and treatment cessation, as indicated by the physician, or disease progression.

\section{Results}

Five patients received CP-B therapy between January, 2010 and December, 2011. The 4 patients who completed the CP-B treatment were included in this study. The patients were males, had histologically confirmed adenocarcinomas and were smokers. The CT pattern of the ILD cases was 'nonUIP' (Table I). None of the patients had ever received any treatment for pneumonitis, such as steroids or anti-fibrotic agents. CP-B was administered for 1-6 cycles. The dose of carboplatin was the area under the curve 5, paclitaxel was $200 \mathrm{mg} / \mathrm{m}^{2}$ and bevacizumab was $15 \mathrm{mg} / \mathrm{kg}$ at treatment initiation. The attending physician modified these doses according to toxicity. Three of the 4 patients received maintenance bevacizumab therapy (Table II). Only 1 patient showed a partial response. Neutropenia was the most frequent adverse event. One patient experienced gut perforation (grade 4) during the first cycle of CP-B and underwent surgery. Thereafter, $\mathrm{CP}$ alone was administered to this patient. No pulmonary toxicity was observed when comparing pre- and post-treatment lung parenchyma CT findings. Treatment was terminated due to disease progression in all the patients, with the exception of 1 patient. The time period to treatment failure was 25-427 days.
Total number of ILD-NSCLC patients administered CP-B. A total of 162 patients (35 with SCLC and 127 with NSCLC) were diagnosed with ILD-LC between January, 2010 and December, 2011 in our Institute. A total of 24 patients with NSCLC were administered anticancer agents, whereas 15 patients (including the 4 patients assessed in the present study) received CP. The Japanese Ministry of Health, Labor and Welfare approved the use of bevacizumab for lung cancer in November 2009. The use of bevacizumab was examined at the end of 2009. Patients without metastatic brain tumors or evidence of thrombosis, hemoptysis or cavity lesions [5 of $9 \mathrm{CP}$ patients $(55.6 \%)$ ] were defined as eligible to receive bevacizumab treatment. The remaining patients were defined as ineligible to receive bevacizumab due to brain metastasis, cavity formation, an easy to bleed tumor or fresh cranial infarction. After 2010, 5 of the remaining $6 \mathrm{CP}$ patients were administered bevacizumab. The rate of bevacizumab use in non-squamous cell NSCLC patients receiving chemotherapy was $41.7 \%$.

\section{Discussion}

Findings of this study have shown that CP-B therapy in ILD-NSCLC patients was not associated with pulmonary toxicity. However, the population in this study appeared to be at a low risk due to the adverse events observed.

The rate of bevacizumab-related pneumonitis has been reported to be $0.19 \%$ in a post-marketing investigation (6). This study did not identify the difference between the effect of bevacizumab compared with that of other agents used in combination with bevacizumab. Bevacizumab-related pneumonia has not yet been reported in lung cancer patients. In colorectal cancer, ILD was found to occur in $3.84 \%$ of patients receiving standard chemotherapy with bevacizumab (7). The ILD patients included in this study were males, 3 of 4 patients were smokers, while 1 patient was diagnosed with pulmonary fibrosis. An additional study reported the case of bevacizumabrelated ILD in a 64-year-old woman (8).

In this study, one patient experienced a severe adverse event (grade 4 gut perforation). More than half of the toxic deaths were found to occur within two treatment cycles in two previously published clinical trials $(4,9)$. These results indicate that ILD-NSCLC patients receiving CP-B treatment should be hospitalized for a minimum of two treatment cycles. Maintenance treatment with bevacizumab was safe for at least 
3 cycles. Therefore, this regimen is considered suitable for the selected ILD-NSCLC patients.

New molecular-targeted drugs are potential future treatment options. BIBF1120 is a multiple-target tyrosine kinase inhibitor that targets vascular endothelial (VEGF), platelet-derived growth factors (PDGF), and fibroblast growth factor receptor (FGFR). Compared with placebo in IPF patients, high-dose BIBF1120 was found to inhibit the decrease in lung function and was associated with fewer acute exacerbations (10). A clinical trial comparing 250 and $150 \mathrm{mg}$ BIBF1120 twice daily in NSCLC patients unresponsive to platinum drugs did not show a difference in PFS (11). However, a study assessing the combination of docetaxel (12) or pemetrexed (13) and BIBF1120 is currently being conducted. Depending on the results of this trial, BIBF1120 may be a key agent for the treatment of advanced ILD-NSCLC.

The present study was limited in size, comprising only four patients, for whom data were collected retrospectively. Additionally, no detailed ILD background was available and UIP as well as non-UIP patterns were evaluated using only CT.

CP-B treatment in patients with ILD-NSCLC did not induce pulmonary toxicity. Although this population appears to be at a low risk, this regimen was identified as an alternative treatment option in such patients. Novel molecular-targeting agents may alter the treatment of ILD-LC patients.

\section{References}

1. Bouros D, Hatzakis K, Labrakis H and Zeibecoglou K: Association of malignancy with diseases causing interstitial pulmonary changes. Chest 121: 1278-1289, 2002

2. Isobe K, Hata Y, Sakamoto S, Takai Y, Shibuya K and Homma S: Clinical characteristics of acute respiratory deterioration in pulmonary fibrosis associated with lung cancer following anticancer therapy. Respirology 15: 88-92, 2010.

3. Minegishi Y, Sudoh J, Kuribayasi H, Mizutani H, Seike M, Azuma A, Yoshimura A, Kudoh S and Gemma A: The safety and efficacy of weekly paclitaxel in combination with carboplatin for advanced non-small cell lung cancer with idiopathic interstitial pneumonias. Lung Cancer 71: 70-74, 2011.

4. Sandler A, Gray R, Perry MC, Brahmer J, Schiller JH, Dowlati A, Lilenbaum R and Johnson DH: Paclitaxel-carboplatin alone or with bevacizumab for non-small cell lung cancer. N Engl J Med 355: 2542-2550, 2006.
5. Therasse P, Arbuck SG, Eisenhauer EA, Wanders J, Kaplan RS, Rubinstein L, Verweij J, Van Glabbeke M, van Oosterom AT, Christian MC and Gwyther SG: New guidelines to evaluate the response to treatment in solid tumors. European Organization for Research and Treatment of Cancer, National Cancer Institute of the United States, National Cancer Institute of Canada. J Natl Cancer Inst 92: 205-216, 2000.

6. Chugai Pharmaceutical Co, Ltd.: http://chugai-pharm.jp/hc/ss/pr/ safe/report/ava/index.html. Accessed July 26, 2012.

7. Usui K, Katou Y, Furushima K, Tanaka Y, Tanai C and Ishihara T: Interstitial lung disease during chemotherapy combined with oxaliplatin and/or bevacizumab in advanced colorectal cancer patients. Jpn J Clin Oncol 41: 498-502, 2011.

8. Tamura J, Nakauchi M, Nakayama Y, Kitaguchi K, Sakikubo M, Ura K, Taira K, Ohe H, Yoshikawa A, Ishigami S and Baba N: A case of interstitial pneumonitis induced by FOLFIRI+bevacizumab combination therapy for liver and lung metastasis of colon cancer. Gan To Kagaku Ryoho 36: 2665-2668, 2009 (In Japanese).

9. Niho S, Kunitoh H, Nokihara H, Horai T, Ichinose Y, Hida T, Yamamoto N, Kawahara M, Shinkai T, Nakagawa K, Matsui K, Negoro S, Yokoyama A, Kudoh S, Kiura K, Mori K, Okamoto H, Sakai H, Takeda K, Yokota S, Saijo N and Fukuoka M; JO19907 Study Group: Randomized phase II study of first-line carboplatin-paclitaxel with or without bevacizumab in Japanese patients with advanced non-squamous non-small cell lung cancer. Lung Cancer 76: 362-367, 2012.

10. Richeldi L, Costabel U, Selman M, Kim DS, Hansell DM, Nicholson AG, Brown KK, Flaherty KR, Noble PW, Raghu G, Brun M, Gupta A, Juhel N, Kluglich M and du Bois RM: Efficacy of a tyrosine kinase inhibitor in idiopathic pulmonary fibrosis. $\mathrm{N}$ Engl J Med 365: 1079-1087, 2011.

11. Reck M, Kaiser R, Eschbach C, Stefanic M, Love J, Gatzemeier U, Stopfer P and von Pawel J: A phase II doubleblind study to investigate efficacy and safety of two doses of the triple angiokinase inhibitor BIBF 1120 in patients with relapsed advanced non-small cell lung cancer. Ann Oncol 22: 1374-1381, 2011.

12. US National Institutes of Health: Lume-Lung 1: BIBF 1120 plus docetaxel as compared to placebo plus docetaxel in 2nd line non-small cell lung cancer. http://clinicaltrials.gov/ct2/show/ NCT00805194. Accessed July 26, 2012.

13. US National Institutes of Health: Lume-Lung 2: BIBF 1120 plus pemetrexed compared to placebo plus pemetrexed in 2 nd line nonsquamous NSCLC. http://clinicaltrials.gov/ct2/show/ NCT00806819. Accessed July 26, 2012. 